\title{
Organogenèse et croissance du maître-brin du blé tendre (Triticum aestivum) du semis à la floraison. I. - Relations observées entre la croissance foliaire et la différenciation des ébauches foliaires ou florales.
}

Pierre MALVOISIN (1)

I.N.R.A., Station d'Amélioration des Plantes, BV 1540, F 21034 Dijon

(1) Adresse actuelle : CIMMYT, Londres 40, Colonia Juarez, Delegation Cuauhtemoc, Apartado postal 6-641, 06600 Mexico D.F.

\begin{abstract}
La morphogenèse du maître brin du blé est décrite sous l'angle des relations qui s'établissent entre l'activité de croissance sous-apicale et l'activité organogène des méristèmes terminaux. En milieu contrôlé, à température constante, la durée séparant l'émergence de deux feuilles successives est fixée précocement et ne subit pas de variations. Cette propriété est conservée en milieu naturel à condition d'indexer cette durée sur la somme des températures moyennes journalières (base $0^{\circ} \mathrm{C}$ ). On appelle thermochrone cette durée élémentaire de croissance. Trois périodes jalonnent le parcours : pendant la période juvénile, l'initiation est régulière au rythme de deux initiums par thermochrone ; à l'apparition des premières ébauches d'entrenœuds, ce rythme augmente jusqu'à la formation de l'épillet terminal (période de transition); puis, au cours de la période florale, l'initiation des fleurs dans chaque épillet se fait à nouveau selon un rythme régulier. On propose en conclusion les bases d'approche d'une étude du fonctionnement intégré de la plante du semis à l'épiaison.
\end{abstract}

Mots clés additionnels : Développement, corrélations, modélisation.
Organogenesis and growth of the main culm of wheat from sowing to flowering. I. Relationships between leaf growth and the differentiation of young leaves or flowers.

The integrated pattern of growth and development of the main culm of wheat (Triticum aestivum L.) from germination to heading stage is described. Under controlled conditions with constant temperature, the time between the appearance of two successive leaves was determined early in plant development and remained constant throughout plant development. Similarly, under natural conditions, this time (called thermochrone) remained constant, depending only on the sum of mean daily temperatures (base $0^{\circ} \mathrm{C}$ ). The activity of the terminal meristems appeared to be strongly influenced by the sub-apical part : during the early stages of growth (juvenile period), the initiation rate of primordia was slow and constant at two primordia per thermochrone ; at the appearance of the first young internodes, the initiation rate increased (transition period) ; when the shoot apical meristem itself became a spikelet (terminal spikelet), the floral progress of spikelet buds returned to a regular rate (floral period). The main features of studying the integrated pattern of wheat morphogenesis are developed.

Additional key words : Development, correlations, modelization.

\section{INTRODUCTION}

Si le fonctionnement du dôme terminal (EVANS \& BERG, 1972), l'évolution des stades phénologiques (JONARD et al., 1952 ; KIRBY \& APPLEYARD, 1981) ou la croissance des organes (WILliAMS, 1975) du blé sont bien connus, peu de recherches portent sur l'étude des fonctions de coordination qui gouvernent le développement et la croissance de cette plante (VINCENT et al., 1977).

Une plante est composée d'un empilement d'organes ; chaque organe suit un programme morphogénétique propre et c'est un jeu de corrélations, lui-même en mouvance, qui assure à l'ensemble un mouvement intégré (NozERAN et al., 1971, 1978) : il apparaît opportun de chercher à mieux comprendre la logique de ce fonctionnement intégré (VINCENT et al., 1977). 
Comme modèle d'étude, le blé offre certains avantages. C'est une espèce autogame qui s'est diversifiée en un grand nombre de génotypes spécialisés quant à leurs adaptations. L'outil génétique se substitue aisément aux manipulations par incision ou décapitation : en comparant les parcours d'organogenèse et de croissance suivis par des couples de lignées isogéniques ou mutantes sur divers caractères affectant la morphogenèse (vernalisation, photopériode), après avoir analysé le déroulement d'un parcours type, on est en mesure de révéler l'invariant qui relève des principes du fonctionnement intégré et qui va faire l'objet de notre propos, du variant qui représente le facteur propre d'adaptation au milieu.

Cette étude comporte quelques restrictions ; seule la $1^{\text {re }}$ partie du cycle de morphogenèse, limitée au développement des parties aériennes du maître-brin est abordée. Dans ce travail, nous présentons la méthode d'observation puis nous traçons le cadre général du déroulement d'un parcours type dans la période considérée.

\section{MÉTHODE D’OBSERVATION}

Etudier une morphogenèse nécessite de prendre des précautions méthodologiques: la terminologie utilisée, la manière même dont sont rapportées les observations font partic du travail d'analyse qui est entrepris.

\section{A. Mesure du stade foliaire en milieu contrôlé : le phyllochrone}

On a utilisé la lignée «Cappelle 239 » mutant alternatif de la lignée d'hiver «Cappelle» (MAIA et al., 1967). Après nettoyage à l'hypochlorite de calcium, un lot de graines a été placé dans une étuve à germination $\left(36 \mathrm{~h}, 20^{\circ} \mathrm{C}\right)$. Puis on a repiqué les semences présentant des coléoptiles sensiblement de même longueur dans 48 pots, à raison de 4 graines par pot ; les pots sont remplis d'un mélange de tourbe-gravier ; ils ont été placés dans une chambre climatisée réglée à $20^{\circ} \mathrm{C}$ continus et $16 \mathrm{~h}$ d'éclairement, pour une intensité de $70 \mathrm{Wm}^{-2}$ environ sur toute la durée de l'expérience. L'arrosage a été effectué régulièrement avec une solution nutritive standard.

L'échelle de notation de l'état de croissance de la plante est basée sur le degré d'avancement de la sortie des feuilles, que nous appelons «stade foliaire». HAUN (1973) propose une échelle dont nous avons gardé le principe mais que nous avons légèrement modifiée. On détermine d'abord le nombre de feuilles pleinement sorties, puis la feuille en expansion est affectée d'une décimale correspondant à son degré d'avancement à partir de son émergence. Un point de couleur (peinture glycérophtalique), à la base des limbes d'ordre 3, 6, 9 permet de repérer le maître-brin et de noter le stade foliaire sans ambiguïté. Les observations de l'avancement du stade foliaire en fonction du temps sont effectuées sur 8 plantes tirées au sort. Un ajustement par régression linéaire est calculé ; le tableau 1 montre que les coefficients de détermination des droites de régression sont tous élevés. On définit ainsi le phyllochrone ou durée séparant l'émergence de 2 feuilles successives. Un test de non-parallélisme appliqué à l'ensemble des régressions montre que les droites ne sont pas parallèles, chaque plante suivant une trajectoire spécifique caractérisée par son phyllochrone ; les droites forment un faisceau (fig. 1), les différences de rythme d'émergence s'inscrivant très tôt, dès les premières notations. Finalement, en calculant la régression linéaire des stades foliaires moyens en fonction du temps, on obtient une valeur moyenne du phyllochrone, caractéristique de cette expérimentation (tabl. 1 ; fig. 2). On note que la répartition des points de part et d'autre de la droite de régression suggère la présence d'un rythme dont la période couvre plusieurs phyllochrones. En conséquence, on doit considérer l'ensemble du parcours pour calculer le phyllochrone. Il reste à s'assurer que l'on retrouve ces propriétés de l'avancement du stade foliaire dans d'autres situations avant d'en tirer les conséquences.

TABLEAU 1

Caractéristiques des droites de régression du stade foliaire en fonction du temps. «Cappelle 239 », $20{ }^{\circ} \mathrm{C}$, $16 \mathrm{~h}$ d'éclairement. a. Si $b$ représente la pente de la droite de régression, le phyllochrone est égal à $1 / b$ (en jours). b. Si a représente l'ordonnée à l'origine, l'émergence théorique de la première feuille vaut - a/b (jours).

The regression lines of leaf appearance on time. 'Cappelle 239', growth room at $20{ }^{\circ} \mathrm{C}$ with $16 \mathrm{~h}$ photoperiod. a. If $b$ is the slope of the regression line, the phyllochrone is equal to $I / b$ (in days). $b$. The value of $y$ for $x=o$, i.e. - a/b, represents the time needed for the emergence of the first leaf (days).

\begin{tabular}{|c|c|c|c|c|c|}
\hline $\mathrm{N}^{\circ}$ plante & $\begin{array}{c}\text { Nombre } \\
\text { de feuilles } \\
\text { final }\end{array}$ & $\begin{array}{l}\text { Phylloa } \\
\text { chrone }\end{array}$ & $\begin{array}{c}\text { Coefficient } \\
\text { détermination }\end{array}$ & $\begin{array}{c}\text { Emergence }^{b} \\
\text { théorique } \\
1^{\text {re }} \text { feuille }\end{array}$ & $\begin{array}{c}\text { Nombre } \\
\text { de jours } \\
\text { semis-épiaison }\end{array}$ \\
\hline 1 & 9 & 6,2 & 0,998 & 1 & 71 lente \\
\hline 2 & 9 & 6,1 & 0,998 & 1 & 70 \\
\hline 3 & 9 & 5,9 & 0,999 & 0,5 & 67 moyenne \\
\hline 4 & 9 & 5,8 & 0,995 & 0,5 & 63 \\
\hline 5 & 9 & 5,7 & 0,992 & 2 & 66 \\
\hline 6 & 9 & 5,6 & 0,998 & 2 & 65 \\
\hline 7 & 9 & 5,5 & 0,997 & 0,5 & 62 rapide \\
\hline 8 & 9 & 5,5 & 0,998 & 1 & 64 \\
\hline $\begin{array}{c}\text { valeurs } \\
\text { moyennes }\end{array}$ & 9 & 5,9 & 0,998 & 1 & 66 \\
\hline
\end{tabular}




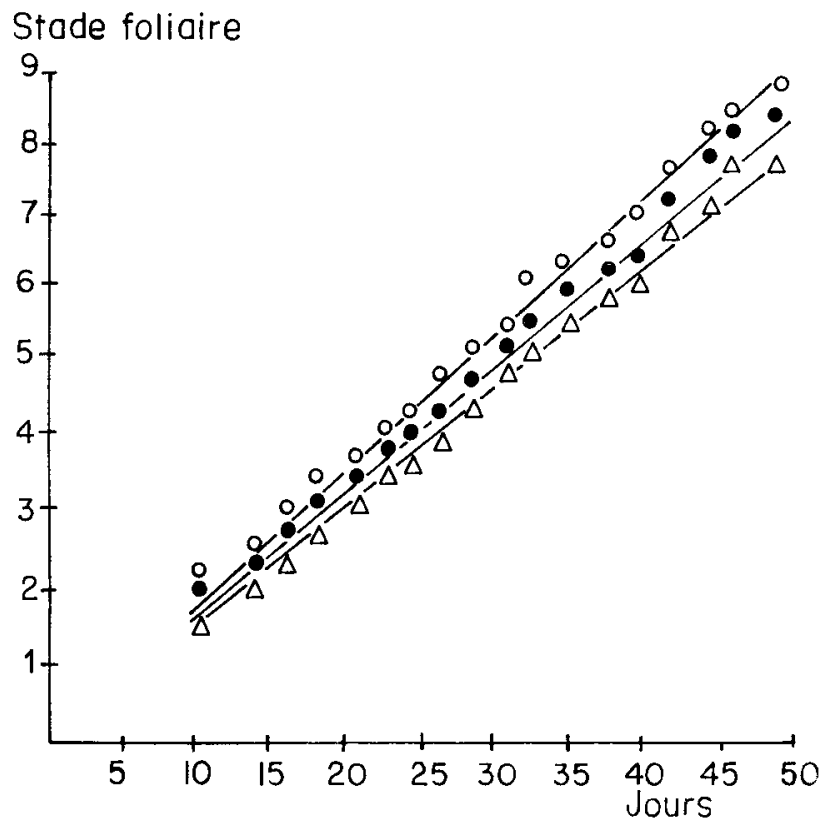

Figure 1

Evolution $d u$ stade foliaire de 3 plantes: $\Delta$ plante "lente», - plante « moyenne », O plante « rapide « «appelle $239 », 20{ }^{\circ} \mathrm{C}$, $16 \mathrm{~h}$. Les paramètres des droites de régression figurent au tableau 1 .

Regression of leaf stages of 3 plants on time : $\Delta$ " slow " plant, - " normal » plant, $\bigcirc$ « fast " plant. 'Cappelle 239', $20^{\circ} \mathrm{C}, 16 \mathrm{~h}$ photoperiod. Parameters of regression lines in table 1.

\section{B. Mesure du stade foliaire en milieu naturel : le thermochrone}

Nous avons semé le 22 novembre 1979, à la Station d'Amélioration des Plantes de Dijon, un essai en blocs complets à 3 répétitions avec un semoir en lignes et de précision, réglé à $17 \mathrm{~cm}$ d'écartement entre les lignes. Cet essai comprenait 6 variétés de précocité différente : «Talent » variété précoce ; 3 lignées demiprécoces désignées par les sigles B 10, B 20A, B 30 ; "Cappelle », une variété demi-tardive ; « Starke », variété tardive. Nous ne rapportons en détail que les résultats concernant "Cappelle », les conclusions qui vont se dégager s'appliquant aux autres cultivars.

A la sortie de l'hiver, la densité de semis était de 140 plantes $/ \mathrm{m}^{2}$. On a suivi l'évolution du stade foliaire de 10 plantes successives dans chacun des blocs ; sur les 30 plantes ainsi notées, 7 ont disparu pendant le tallage et la montaison et ont été écartées de l'étude. Sur les 23 restantes qui ont épié, les 2/3 avaient 12 feuilles (sous-groupe majoritaire), le tiers restant 11 feuilles (sous-groupe minoritaire).

On calcule le phyllochrone moyen de chacun des sous-groupes, non pas en fonction du nombre de jours depuis le semis mais en fonction de la somme des températures moyennes journalières (base $0{ }^{\circ} \mathrm{C}$ ). Dans ce cas, l'ajustement linéaire s'avère excellent (tabl. 2). La régularité de l'avancement du stade foliaire se confirme ; en outre, on met en évidence l'influence de la température : il existe donc au sein de la plante une activité, contrôlée par la température, qui coordonne la croissance des organes de manière régulière tout au long du parcours et, comme on a tout lieu de supposer que l'émergence successive des

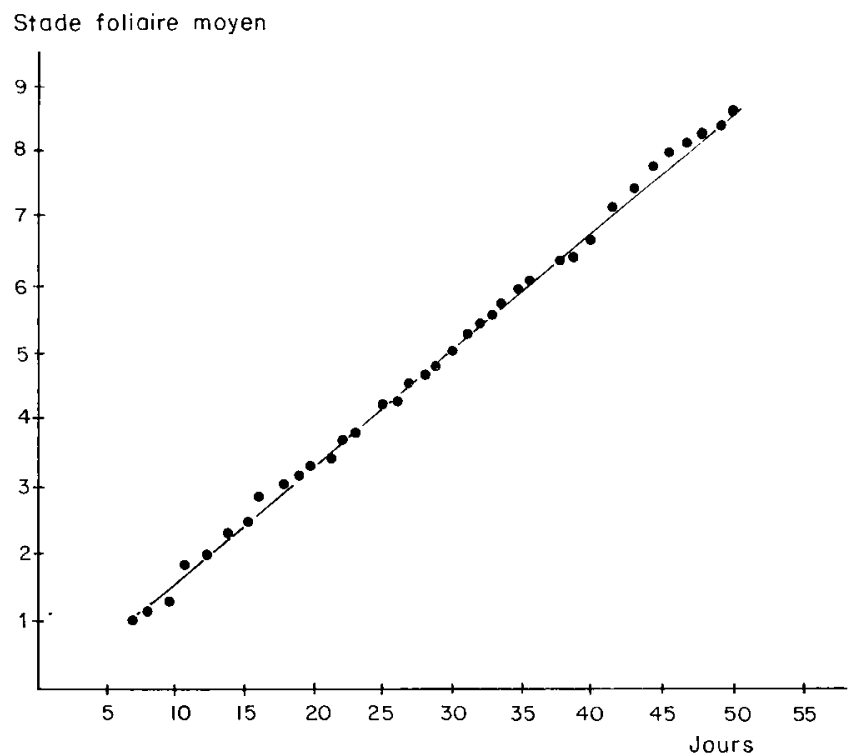

Figure 2

Evolution du stade foliaire en fonction du temps; "Cappelle 239 " moyenne de 8 plantes ; les paramètres de la droite de régression figurent tableau 1.

Regression of leaf stages on time ; 'Cappelle 239'; mean values of 8 plants; parameters of regression line in table 1.

feuilles n'est qu'une manifestation particulière de cette activité, nous abandonnons le terme de phyllochrone pour celui de thermochrone qui a une portée plus générale et qui souligne le rôle joué par la température.

\section{Propriétés associées au thermochrone}

Un examen attentif des tableaux 2 et 3 montre qu'à dates d'épiaison identiques, les plantes à 11 feuilles ont tendance à avoir un thermochrone plus important que les plantes à 12 feuilles. Cette tendance est visible dès les premières notations. Cette observation est confirmée sur les autres cultivars expérimentés, le nombre final de feuilles variant cependant avec la précocité (tabl. 4) ; ainsi la valeur du thermochrone semble s'établir précocement et se conserve, en moyenne, tout au long du parcours. Une autre manipulation (MALVOISIN, 1981) vient étayer ce point de vue : nous avons procédé pendant 2 années consécutives à des

\section{TABLEAU 2}

Comparaison des thermochrones des 2 sous-populations «Cappelle », milieu naturel.

Comparison of the thermochrones of the 2 sub-populations. 'Cappelle', field conditions.

\begin{tabular}{lcc}
\hline \hline & $\begin{array}{c}\text { Plantes à } \\
11 \text { feuilles } \\
\text { (minoritaire) }\end{array}$ & $\begin{array}{c}\text { Plantes à } \\
12 \text { feuilles } \\
\text { (majoritaire) }\end{array}$ \\
\hline $\begin{array}{l}\text { Thermochrone } \\
\text { en degrés/jours }\end{array}$ & 83 & 79 \\
$\begin{array}{l}\text { Coefficient de } \\
\text { détermination }\end{array}$ & 0,988 & 0,993 \\
Date d'épiaison & $8 / 6$ & $8 / 6$ \\
\hline \hline
\end{tabular}


TABLEAU 3

Comparaison des stades foliaires moyens des 2 sous-populations. "Cappelle ", milieu naturel.

Comparison of the leaf stages of the 2 sub-populations. 'Cappelle', field conditions.

\begin{tabular}{|c|c|c|}
\hline \multirow[b]{2}{*}{ Date } & \multicolumn{2}{|c|}{ Stade foliaire moyen } \\
\hline & $\begin{array}{l}\text { Sous-population } \\
\text { minoritaire à } \\
11 \text { feuilles }\end{array}$ & $\begin{array}{l}\text { Sous-population } \\
\text { majoritaire à } \\
12 \text { feuilles }\end{array}$ \\
\hline $13 / 2$ & 1,99 & 1,99 \\
\hline $27 / 2$ & 2,84 & 2,93 \\
\hline $12 / 3$ & 3,76 & 3,81 \\
\hline $24 / 3$ & 4,58 & 4,59 \\
\hline $3 / 4$ & 5,88 & 5,91 \\
\hline $14 / 4$ & 6,89 & 6,95 \\
\hline $23 / 4$ & 7,68 & 7,95 \\
\hline $6 / 5$ & 8,91 & 9,23 \\
\hline $15 / 5$ & 9,95 & 10,43 \\
\hline $22 / 5$ & 10,73 & 11,31 \\
\hline
\end{tabular}

TABLEAU 4

Thermochrones des différentes sous-populations de quatre cultivars. Milieu naturel.

a. Thermochrone en degrés-jours.

b. Nombre final de feuilles de la sous-population.

Thermochrones of the subpopulations of four cultivars. Field conditions.

a. Thermochrone in degree-days.

$b$. Final number of leaves in the subpopulation.

\begin{tabular}{lllllllll}
\hline \hline Variété & \multicolumn{1}{c}{$\begin{array}{c}\text { Type de } \\
\text { précocité }\end{array}$} & Lente & $\begin{array}{c}\text { Sous- } \\
\text { population } \\
\text { moyenne } \\
\text { majoritaire }\end{array}$ & Rapide \\
& & & a & b & a & b & a & b \\
\hline Talent & précoce & & & 77 & 10 & 75 & 11 \\
B30 & $1 / 2$ préc. & 83 & 10 & 78 & 11 & & \\
Cappelle & $1 / 2$ tard. & 83 & 11 & 79 & 12 & & \\
Starke & tardive & & & 79 & 12 & 74 & 14 \\
& & & & & & et 13 & & \\
\hline \hline
\end{tabular}

repiquages en serre de la lignée $\mathrm{B} 30$ dans des conditions identiques d'une année sur l'autre $\left(20^{\circ} \mathrm{C}\right.$, jours continus), au même stade phénologique des plantes. Les résultats présentés au tableau 5 sont nets : En 1978/1979, le thermochrone au champ valait $92^{\circ} \mathrm{C}$ (semis précoce); l'année suivante, seulement $78^{\circ} \mathrm{C}$ (semis tardif); la valeur du thermochrone est donc bien spécifique de chaque expérimentation. De plus, cette différence se conserve même après un repiquage qui met les plantes pourtant dans des conditions de milieu sensiblement identiques.

Certains paramètres qui conditionnent la valeur du thermochrone s'établissent précocement et se conservent tout au long du parcours : il s'agit d'un effet « milieu » intégrant vraisemblablement les conditions de levée et la qualité de la semence.

Quels que soient les mécanismes mis en cause, cette propriété remarquable du thermochrone est à la base d'une méthode pratique d'observation permettant de comparer avec précision n'importe quels parcours.
TABLEAU 5

Comparaison des thermochrones (en degrés-jours) du cultivar B30 en milieu naturel et après repiquage en serre $\left(20^{\circ} \mathrm{C}\right.$, jours continus) sur deux années.

Comparison of thermochrones (in degree-days) of cultivar B30 in field conditions and after transplanting into the glasshouse $\left(20{ }^{\circ} \mathrm{C}\right.$, continuous light). Two years of experiments.

\begin{tabular}{|c|c|c|}
\hline & \multicolumn{2}{|c|}{ Année } \\
\hline & $1978 / 1979$ & $1979 / 1980$ \\
\hline & \multicolumn{2}{|c|}{ milieu naturel } \\
\hline Date de semis & 25 octobre & 22 novembre \\
\hline Thermochrone & 92 & 78 \\
\hline \multirow[t]{2}{*}{ Nb. final de feuilles } & 11 & 11 \\
\hline & \multicolumn{2}{|c|}{$1^{\text {er }}$ repiquage (mars) } \\
\hline $\begin{array}{l}\text { Stade foliaire } \\
\text { au repiquage }\end{array}$ & 4,7 stade $\mathrm{Al}$ & 4,7 stade Al \\
\hline Thermochrone & 96 & 76 \\
\hline \multirow[t]{2}{*}{$\mathrm{Nb}$. final de feuilles } & 10 & 10 \\
\hline & \multicolumn{2}{|c|}{$2^{\mathrm{e}}$ repiquage (avril) } \\
\hline $\begin{array}{l}\text { Stade foliaire } \\
\text { au repiquage }\end{array}$ & 7,1 stade $\mathrm{C} 12$ & 7,0 stade $\mathrm{C} 12$ \\
\hline Thermochrone & 88 & 70 \\
\hline $\mathrm{Nb}$. final de feuilles & 11 & 11 \\
\hline
\end{tabular}

\section{Méthode et technique d'observation}

Cette méthode repose sur les 4 règles suivantes :

1. A chaque instant, l'âge biologique d'une plante est assimilé à son stade foliaire ;

2. Un lot de plantes de même nombre final de feuilles, soumis à des conditions de milieu identiques, présente une hétérogénéité de stade foliaire. Cette hétérogénéité s'accroît avec le temps, mais reste limitée. Un nombre modeste de plantes témoins, choisies au hasard dans la population, suffit à donner une bonne estimation de la moyenne de la population. Le passage du temps biologique (exprimé en "stade foliaire ») au temps calendaire est donné par la régression du stade foliaire moyen des plantes témoins en fonction de la somme des températures moyennes journalières (base $0{ }^{\circ} \mathrm{C}$ ) ; cette régression permet de calculer le thermochrone ;

3. A intervalles de temps réguliers, on prélève 2 ou 3 plantes dont le stade foliaire se rapproche du stade foliaire moyen des plantes témoins pour effectuer les mesures désirées. On rapporte selon l'échelle de temps biologique, c'est-à-dire en stade foliaire, les mesures individuelles ;

4. Enfin, en milieu contrôlé comme au champ, il apparaît parfois un lot minoritaire de plantes que l'on qualifie de « déficientes ». Les plantes qui constituent cette sous-population peuvent avoir un thermochrone qui s'écarte notablement du thermochrone moyen ; leur aspect est différent de la moyenne, parfois elles n'atteignent pas l'épiaison. Ce type de plantes a été écarté de notre étude.

A l'aide de cette méthode, un nombre limité de plantes suffit à décrire entièrement un parcours d'organogenèse et de croissance : on découpe, selon le plan médian longitudinal du maître-brin choisi, une tranche fine de moins d'1 mm d'épaisseur laissant 
intact l'apex. La préparation, montée sur une lame, est observée immédiatement à la loupe binoculaire ou au microscope. On peut conserver certaines préparations dans du baume du Canada, après fixation au carmin acétique (quelques secondes).

\section{CADRE DU PARCOURS SEMIS-ÉPIAISON}

Si la croissance sous-apicale, mesurée par l'avancement du stade foliaire, se déroule régulièrement tout au long du parcours, il n'en est pas de même de l'activité organogène apicale.

\section{A. Notion de phytomère}

La structure du dôme apical a été décrite par SHARMAN (1945), EVANS \& BERG (1972) et WILLIAMS (1975). Le dôme apical, selon la conception de SCHMIDT généralement admise, se compose du corpus (noté C), massif cellulaire profond à cloisonnement non préférentiel et de la tunica, enveloppe extérieure à plusieurs assises (layers), notées L1, L2, L3, en les numérotant de la périphérie vers l'intérieur. Les premiers événements à l'origine de l'apparition d'un initium sont 3 divisions périclines dans la L1, 1 à 2 dans la L2, aucune dans la L3. Les divisions se poursuivent et l'initium apparaît sous forme d'une saillie; en même temps que cet initium se construit, le dôme se soulève par divisions des cellules du corpus et de la tunica et rapidement un autre foyer de divisions périclines apparaît du côté opposé à l'initium formé. Ainsi se mettent en place des massifs cellulaires périphériques et internes qui ont pour particularité d'avoir été initiés pendant la même période de temps. Nous proposons d'appeler cet ensemble phytomère quel que soit son devenir, par extension au sens donné par ETTER (cité par FISCHER, 1973). Si le phytomère évolue en un ensemble limbe, gaine, noud (porteur de la feuille) et éventuellement entrenœud (situé sous le nœud), nous aurons affaire à un phytomère "végétatif »"; si le phytomère évolue en un ensemble épillet, nœud de rachis et entrenœud de rachis, à un phytomère « floral ». A l'épiaison, le nombre de phytomères végétatifs correspond au nombre final de feuilles et l'empilement des phytomères floraux représente l'épi. Nous affectons un numéro de position à chaque phytomère selon son ordre d'apparition, le $1^{\text {er }}$ phytomère correspond à la $1^{\text {re }}$ feuille. On peut alors effectuer un décompte des initiums créés pour mesurer le rythme d'initiation par thermochrone. Enfin on note l'avancement floral des épillets sur un des épillets les plus avancés du secteur médian de l'épi, selon une échelle inspirée de la description de JONARD et al. (1952) (annexe 1).

\section{B. Les trois périodes juvénile, transitoire et florale du parcours}

La figure 3 montre l'évolution du nombre d'initiums en fonction du temps. A la vue de la disposition des points, on remarque une rupture nette du taux d'initiation au stade Al, à l'apparition des premières ébauches d'entrenœuds, et un arrêt de l'initiation au stade C12 de l'épillet de référence. Le parcours est scindé en 3 périodes :
1) Une $1^{\text {re }}$ période, au rythme d'initiation lent, du semis au stade Al, que nous qualifions de « période juvénile ». C'est pendant cette période que se créent les phytomères végétatifs ( 3 phytomères sont cependant déjà présents dans l'embryon).

2) Une $2^{\mathrm{e}}$ période, au rythme d'initiation rapide, qui se termine par l'initiation du dernier phytomère, correspondant au futur épillet terminal. Pendant cette période, que nous appelons «période transitoire», se mettent en place les phytomères floraux qui forment l'épi. Cette période est aussi celle du tallage herbacé de la plante.

3) Enfin une dernière période, la « période florale ", où chaque bourgeon évolue floralement jusqu'à la fécondation. Cette période couvre la montaison.

Si l'on veut quantifier les taux d'initiation pendant les périodes juvénile et transitoire, on calcule 2 régressions linéaires, l'une ajustant l'évolution du nombre d'initiums en fonction du stade foliaire du semis au stade A1, l'autre du stade A1 au stade C12, la disposition des points s'y prêtant particulièrement. On obtient une valeur moyenne de la vitesse d'initiation pendant les périodes considérées (fig. 3).

\section{Corrélations croissance sous-apicale-activité apicale}

Une remarque importante se dégage de l'examen de la figure 3. En effet, on constate que l'activité d'initiation est régulière pendant la période juvénile : on compte environ 2 initiums créés par thermochrone, autrement dit, il se crée 2 fois plus de phytomères qu'il n'apparaît de feuilles. A l'amorce de la période transitoire, au moment où apparaissent les premières ébauches d'entrenœud, ce taux augmente brutalement; il devient en moyenne 3 à 4 fois supérieur à ce qu'il était auparavant. Enfin on semble revenir à une régularité d'initiation pendant la période florale avec la réalisation d'un pas floral (voir annexe 1), dans tous les épillets de l'épi, par thermochrone.

Ces faits expérimentaux, sans constituer de preuves, suggèrent la présence d'une relation étroite entre la croissance sous-apicale et l'activité organogène apicale.

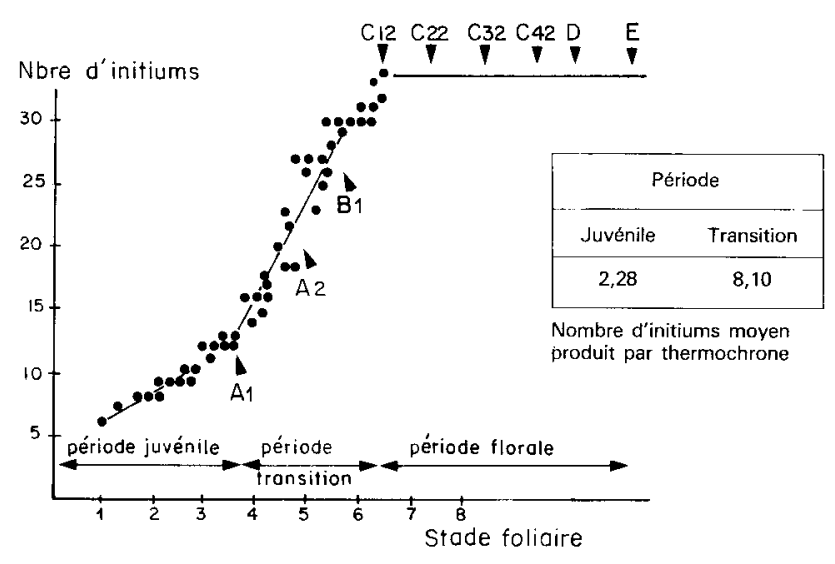

Figure 3

Evolution du nombre d'initiums en fonction du temps. Les 3 périodes du parcours du blé; $A 1, A 2 \ldots$ voir annexe 1.

Increase in primordium production with time. Three periods of wheat growth and development. A1, A2... see appendix 1. 


\section{DISCUSSION}

Notre réflexion sur le déroulement de l'organogenèse et de la croissance du blé s'articule autour des 2 points suivarits : le $1^{\text {er }}$ porte sur le fait que le parcours de la plante s'assimile à un parcours par étapes, la dimension d'une étape étant donnée par le thermochrone, le nombre d'étapes par le nombre de phytomères végétatifs qui se différencieront; le $2^{\mathrm{e}}$ concerne la nature des relations qui s'établissent entre l'activité organogène apicale et la croissance sousapicale.

La linéarité de l'avancement du stade foliaire en fonction de la somme des températures moyennes journalières (base $0^{\circ} \mathrm{C}$ ) cumulées depuis le semis est signalée par GALlaGHER (1979), DELECOLlE \& GuRNADE (1980) pour ne citer que ces 2 auteurs. L'avancement régulier du stade foliaire manifeste la présence d'une activité de croissance parfaitement coordonnée à la fois à l'intérieur des phytomères et entre eux. Le thermochrone mesure l'étendue thermique d'une étape élémentaire de croissance, étendue qui intègre probablement aussi bien la température au niveau du système aérien qu'au niveau du système radiculaire (Chaillou, 1976, sur maïs). Ce qui nous semble remarquable c'est que la valeur de thermochrone est déterminée précocément et reste stable, en moyenne, tout au long du parcours, insensible aux variations qui affectent l'activité organogène apicale ; cette valeur, par contre, varie d'une expérimentation à l'autre : l'observation d'un grand nombre de situations indique une valeur moyenne de $100{ }^{\circ} \mathrm{C}$, avec des écarts possibles de $25{ }^{\circ} \mathrm{C}$ de part et d'autre de cette moyenne (MALVOISIN, 1981).

Si l'étendue d'une étape élémentaire de croissance reste stable quelle que soit l'activité apicale, le nombre d'étapes par contre, fonction du nombre de phytomères qui évolueront en phytomères végétatifs (nombre final de feuilles), dépend des conditions de milieu qui déterminent l'évocation florale (BERNIER, 1971) puis l'initiation florale pendant la période de transition. Cette influence du milieu est à raisonner en erme d'interaction entre l'état de vernalisation et la sensibilité à la photopériode.

$\mathrm{Du}$ point de vue des applications pratiques, nous pensons, comme Delecolle \& GuRNADE (1980), qu'il n'y a pas de loi thermique généralisable à rechercher dans le déclenchement et la fin de la transition ; toutefois, si on raisonne sur un génotype donné semé à la même époque dans des conditions de milieu identiques, on vérifie que le déroulement du parcours est sensiblement le même d'une année à l'autre (surtout en culture de printemps) : en utilisant les sommes de températures pour estimer des dates d'apparition de stades critiques, on ne fait qu'utiliser ces propriétés de la plante que nous avons mis en évidence. Cependant, pour une culture d'hiver, il nous paraît indispensable d'étalonner chaque année le déroulement du parcours par une observation in situ du début et de la fin de la période de transition.

Le $2^{e}$ élément de réflexion concerne les relations qui s'établissent entre l'activité de croissance sous-apicale, matérialisée par l'avancement régulier du stade foliaire, et l'activité organogène apicale.

L'activité du dôme apical a fait l'objet de nom- breux travaux discutés par STERN \& KIRBY (1979). Pour notre part, nous soulignons les faits suivants :

1. Régularité de l'initiation pendant la période juvénile au rythme de 2 initiums par thermochrone; GRIGNAC (1965) a fait cette observation sur Triticum durum.

2. Au moment de l'apparition des premières ébauches d'entrenœud, le taux d'initiation augmente (stade A1) (JONARD et al., 1952).

3. Retour à une régularité dans l'avancement floral des épillets pendant la période florale, avec la réalisation d'un pas floral (annexe 1) dans tous les épillets, par thermochrone. Cette régularité se manifeste nettement si on reprend les nombreuses observations de stades de développement rapportées par MASLEMEYNARD (1980). En réalité 2 questions différentes sont soulevées : on fait l'hypothèse, pendant la période juvénile et florale, d'une relation du type « relation d'asservissement 》 de la zone sous-apicale sur l'activité des méristèmes terminaux (dôme terminal puis population de bourgeons d'épillets) ; par contre, pendant la période de transition, c'est un problème de compétence des phytomères néoformés qui est mis en cause. Ces questions sont complexes : elles font l'objet d'un débat entre morphogénéticiens (NOZERAN et al., 1971 ; HALPERIN, 1978).

\section{CONCLUSION}

La figure 4 schématise le cadre dans lequel se déroule un parcours semis-épiaison. Trois informations seulement sont utilisées pour en tracer avec pré-


Figure 4

Presentation schématique d'un parcours semis-épiaison. $A 1, A 2 \ldots$ voir annexe 1.

Schematic presentation of the growth and development pattern of wheat from seeding to heading stages. A1, A2... see appendix 1 . 
cision le contour :

1. La valeur du thermochrone, défini comme la durée, exprimée en nombre de degrés-jours, nécessaire à l'apparition de 2 feuilles successives.

2. La date de semis (S) et les dates d'apparition des stades Al (premières ébauches d'entrenœud), C (épillet terminal) et $\mathrm{E}$ (épiaison), qui permettent de scinder le parcours en 3 périodes : juvénile (S-A1), transitoire (A1-C), florale (C-E).

3. Le nombre final de feuilles de la sous-population majoritaire de plantes.

Notre expérience nous indique qu'un nombre restreint de plantes suffit pour décrire de cette manière le parcours avec précision, à condition de prendre soin de repérer la sortie des feuilles sur des plantes témoins représentatives de la population étudiée.

Régularité du fonctionnement de la croissance sousapicale et relations étroites entre cette croissance et l'activité apicale sont les aspects nisarquants du déroulement du parcours : celui-ci s'effectue étape par étape, la dimension d'une étape, calculée par le thermochrone, est fixée précocement et ne subit pas de variations. Le nombre d'étapes est donné par le nombre final de feuilles.

Nous avons mis en évidence des relations étroites entre l'activité de croissance sous-apicale, matérialisée par l'avancement régulier du stade foliaire, et l'activité organogène apicale selon des modalités propres à chacune des 3 périodes. En préalable à toute approche de modélisation, il apparaît nécessaire d'étudier plus en détail les caractéristiques de cette activité qui coordonne la croissance et semble asservir l'activité apicale.

Quels que soient les mécanismes physiologiques mis en cause, le déroulement de l'organogenèse et de la croissance du blé, dans cette $1^{\text {re }}$ partie du cycle qui couvre la mise en place des organes végétatifs et de l'inflorescence, apparaît comme celui d'un système intégré, dont la logique nous semble abordable par des méthodes d'observation et d'analyse simples.

Reçu le 26 août 1982. Accepté le 29 février 1984.

\section{REMERCIEMENTS}

Ce travail a été réalisé à la Station d'Amélioration des Plantes de Dijon (I.N.R.A.), avec l'appui financier de l'Institut Technique des Céréales et Fourrages (I.T.C.F.).

\section{ANNEXE 1}

Echelle de notation des stades de développement. Les notations de A2 à D sont effectuées sur un des épillets les plus avancés de l'épi, modifiée d'après JONARD et al. (1952).

Growth of the wheat plant. Scores from $A 2$ to $D$ were made on one of the most advanced spikelets. Modified after JONARD et al. (1952).

S Semis.

Sowing.

Al Apparition des premières ébauches d'entre-nœeuds. Appearance of the first internodes.

A2 Apparition du bourgeon d'épillet, stade double ride avancé. Advanced double ridge stage.

BI Apparition de la glume. Glume appearance.

B2 Apparition de la glumelle. Lemma appearance.

C01 Apparition du ler bourgeon floral. First floral bud appearance.

C02 Apparition du $2^{e}$ bourgeon floral. Second floral bud appearance.

C11 3 ébauches d'anthères sur la l're fleur du ler étage. 3 stamen initials on the first flower bud of the first level.

C12 3 ébauches d'anthères sur la $2^{e}$ fleur du $1^{e r}$ étage. 3 stamen initials on the second flower bud of the the first level. On compte les fleurs par étage, chaque étage étant composé de 2 fleurs. De $\mathrm{CO} 2$ à $\mathrm{C12}, \mathrm{Cl} 2$ à $\mathrm{C} 22$, etc., il y a réalisation d'un pas floral, c'est-à-dire la création d'une paire de bourgeons floraux (un étage) par l'apex d'épillet avec simultanément une évolution florale de la paire précédente par la formation de 3 ébauches d'anthères sur les bourgeons floraux.

We used to count the flower buds level by level, 2 flower buds compose one level. From $\mathrm{C02}$ to $\mathrm{Cl2}, \mathrm{Cl2}$ to $\mathrm{C22}$ etc... we get a floral step: the initiation of 2 flower buds (a level) by the terminal spikelet meristem, and at the same time the initiation of 3 stamen initials at both the flower buds of the previous level.

$D$ Gamétogenèse mâle. Stade tétrade. Male gametogenesis. Tetrad stage.

E Epiaison. L'épi dépasse d'un cm de la dernière gaine. Heading stage. One $\mathrm{cm}$ of the spike visible above the last sheath.

\section{RÉFÉRENCES BIBLIOGRAPHIQUES}

Bernier G., 1971. Structural and metabolic changes in the shoot apex in transition to flowering. Can. J. Bot., 49, 803-819.

Chaillou S., 1976. Effet de températures basses appliquées au niveau des racines. Cas du maïs. Rapport CNRS, Gif-sur-Yvette, 29 p.

Delecolle R., Gurnade J. C., 1980. Liaisons entre le développement et la morphologie du blé tendre d'hiver. I. Stades de développement de l'apex, apparition des feuilles et croissance de la tige. Ann. Amélior. Plant., 30 (4), 479-498.

Evans L. S., Berg A. R., 1972. Early histogenesis and semi quanti tative histochemistry of leaf initiation in Triticum aestivum. Am. J. Bot., 59 (10), 973-980.

Fischer J. E., 1973. Developmental morphology of the inflorescence in hexaploid wheat cultivar with and without the cultivar Norin 10 in their ancestry. Can. J. Plant Sci., 53, 7-15.

Gallagher J. N., 1979. Field studies of cereal leaf growth. I. Initia- tion and expansion in relation to temperature and ontogeny. $J$. Exp. Bot., 30 (117), 625-636.

Grignac P., 1965. Contribution à l'étude de Triticum durum. Thèse Doct. Sci. Nat. Fac. Sci. Toulouse.

Halperin W., 1978. Organogenesis at the shoot apex. Annu. Rev. Physiol., 29, 239-262.

Haun J. R., 1973. Visual quantification of wheat development Agron. J., 65, 116-119.

Jonard P., Koller J., Vincent A., 1952. Evolution de la tige et de l'épi chez la variété de blé Vilmorin 27 au cours de la période de reproduction. Ann. Amél. Plant., I (3), 256-276.

Kirby E. J. M., Appleyard M., 1981. Cereal development guide. Cereal unit, NAC, England.

Maia N., Dommergues P., Gillot J., Goujon C., Touvin H., 1967. Etude de mutants de "type de développement " isolés dans des variétés de blé tendre d'hiver. Ann. Amél. Plant., 17 (2), 195-206. 
Malvoisin P., 1981. Le déroulement de l'organogenèse et de la croissance du maître-brin du blé (Triticum aestivum L.). Thèse Doct.-Ing. Univ. de Paris-Sud, Orsay, $\mathrm{n}^{\circ}$ d'ordre 466.

Masle-Meynard J.. 1980. L'élaboration du nombre d'épis chez le blé d'hiver. Influences de différentes caractéristiques de la structure du peuplement sur l'utilisation de l'azote et de la lumière. Thèse Doct.-Ing., INA PG, Paris.

Nozeran R., 1978. Réflexions sur les enchaînements de fonctionnement au cours du cycle des végétaux supérieurs. Bull. Soc. Bot. Fr., 125, 263-280.

Nozeran R., Bancilhon L., Neville P., 1971, Intervention of internal correlations in the morphogenesis of higher plants. Adv. in Morphog., 9, 1-65.
Sharman B. C., 1945. Leaf and bud initiation in the Gramineac. Bot. Gaz., 106, 269-289.

Stern W. R., Kirby E. J. M., 1979. Primordium initiation at the shoot apex in four contrasting varieties of spring wheat in response to sowing date. J. Agric. Sci., Camb., 93, 203-215.

Vincent A., Farcy E., Suty L., 1977. Sur la logique du blé. Doc. ronéoté, I.N.R.A., Station d'Amélioration des Plantes de Dijon.

Williams R. F., 1975. The shoot apex and leaf growth. Camb. Univ. Press, 256 p. 